\title{
Experiência da Unimed Recife no tratamento de 1.039 pacientes com Covid-19
}

\author{
Marcelo Moraes Valença ${ }^{1,2}$ (D) Martha Maria Romeiro Figueiroa F. Fonseca² (iD Cátia Arcuri Branco \\ Alex Maurício Garcia Santos ${ }^{2}$ (iD Antonio Oliveira ${ }^{2}$ (iD) Danilo Ferreira Nunes ${ }^{2}$ (D) Ana Carolina Chiappetta \\ Correia de Araújo² ${ }^{2}$ Fernando Cruz $^{2}$
}

${ }^{1}$ Universidade Federal de Pernambuco, Recife, Pernambuco Brasil; ${ }^{2}$ Unimed Recife, Recife, Pernambuco, Brasil

\section{$\triangle$}

Marcelo Moraes Valença Unimed Recife, Recife, Pernambuco, Brazil +5581999229394 mmvalenca@yahoo.com.br

Este artigo foi editado por: Juliana Ramos de Andrade
Palavras-chave:

Covid-19

Mortalidade

Diabete Melito

Obesidade

\section{Resumo}

Objetivo Descrever dados relacionados com pacientes com Covid-19 internados nos hospitais da Unimed Recife, avaliando dados demográficos, letalidade, uso de ventilador mecânico, presença de doenças associadas, uso da UTI, entre outros aspectos relacionados com o prognóstico desses pacientes.

Método Os dados foram coletados na plataforma de gestão em saúde DRG Brasil, incluindo o período desde 26 de marco de 2020, quando o primeiro paciente com Covid-19 foi internado no Hospital da Unimed III, até o dia 31 de janeiro de 2021. Todos os pacientes que foram internados em um dos três hospitais da Unimed Recife - Hospital Unimed I, Hospital Unimed III e Hospital Geral Unimed Recife (HGMI) - entraram no estudo. Nesse mesmo período avaliamos o número de pacientes com Covid-19 ou suspeita de Covid-19 que foram atendidos na emergência do Hospital Unimed Recife III.

Resultados Foram atendidos 126,553 pacientes na Unidade de Emergência do Hospital Unimed Recife III no período entre 26 de março de 2020 e 31 de janeiro de 2021, desses pacientes atendidos na emergência 39,340 (31,09\%) pacientes foram diagnosticados com tendo Covid-19 ou suspeita de Covid-19. No período de 10 meses foram internados 1.039 pacientes com Covid-19, 61\% com hipertensão, 31,1\% com SARS, 30,0\% com diabete melito e 9,9\% eram obesos. A média de permanência no hospital foi de 11,2 dias. Foram internados na UTI 342/1.039 (32,9\%) pacientes e 57,9\% deles utilizaram ventilação mecânica. A letalidade geral foi de 13,76\% (143 mortes/1.039). Houve um aumento na letalidade por Covid-19 com o aumento da idade. A letalidade no primeiro período da pandemia por Covid-19 foi significativamente maior quando comparado com os últimos meses da pandemia $(17,6 \%$ versus $9,7 \%$, $p<0,001)$. Obesidade aumentou significativamente a letalidade nos pacientes com Covid-19 [120 mortes/ 1.016 pacientes não obesos $(11,8 \%)$ versus 23 mortes/103 pacientes obesos (22,3\%), OR 2,15 (1,30 - 3,50), $p=0,005)]$.

Conclusão Concluímos que a Covid-19 é uma doença com prognóstico pior nos pacientes idosos e com obesidade. Na segunda fase da pandemia por Covid-19 percebemos uma queda significativa na letalidade por Covid-19 nos pacientes internados. Covid-19 é uma doença nova, ainda pouco conhecida, e nos primeiros meses da pandemia não se entendia muito bem sobre os mecanismos pelos quais o vírus se multiplicava nem como o processo fisiopatológico ocorria no organismo infectado.

Este artigo também está publicado em inglês (https://doi.org/10.535435/AvanMed.4) 


\section{Introdução}

- m dezembro de 2019 uma nova doença viral humana sur- giu em Wuhan, China, e foi denominada como Covid-19 ('CO' significa corona, 'VI' vírus e 'D' significa "disease" ou doença; 19 de "2019 novel coronavirus"). Poucos meses depois, os primeiros casos de pacientes com Covid-19 chegam ao Recife. ' Foi um casal que retornava da Europa e chegou no Aeroporto Internacional do Recife/Guararapes no dia 29 de fevereiro de 2020. O primeiro paciente diagnosticado como Covid-19 na América do Sul foi um homem, de 61 anos de idade, que chegou em São Paulo no dia 26 de fevereiro de 2021, de uma viagem com passagem pela Itália. ${ }^{\text {? }}$

Apesar dos cuidados de prevenção houve um crescimento exponencial no número de casos no estado de Pernambuco e no Brasil. ${ }^{2}$ Apenas nos hospitais da Unimed Recife durante o ano de 2020 foram 894 pacientes internados com Covid-19, eram indivíduos com quadro clínico mais grave que justificou o internamento.

Algumas doenças associadas em pacientes com Covid-19 determinam um pior prognóstico, como obesidade ${ }^{3.6}$, uso de inibidores do sistema renina-angioten $\sin ^{7}$, hipertensão $\operatorname{arteria}^{8,9}$, diabete melito ${ }^{5,9}$, idade mais avançada ${ }^{7}$ 0.14, sexo masculino ${ }^{13-16}$, doença pulmonar obstrutiva crônica ${ }^{7}$ e doença renal crônica ${ }^{7}$. Os seguintes parâmetros laboratoriais também estão associados com o prognóstico dos pacientes com Covid-19: linfopenia, níveis elevados de proteína $C$ reativa, contagem de neutrófilos, interleucina-6, D-dímero, desidrogenase láctica e troponina I. 15, 17, 18

Neste artigo os autores pretendem descrever dados relacionados com o tratamento desses pacientes que foram internados nos hospitais da Unimed Recife no ano de 2020 e início de 2021, avaliando dados demográficos, letalidade, uso de ventilador mecânico, presença de afecções que modificam o prognóstico da doença (e.g., diabetes, obesidade), necessidade de internamento na Unidade de Terapia Intensiva (UTI), entre outros aspectos relacionados com o prognóstico desses pacientes.

\section{Método}

Os dados foram coletados na plataforma de gestão em saúde DRG Brasil, incluindo o período desde 26 de março de 2020, quando o primeiro paciente com Covid-19 foi internado no Hospital da Unimed III, até o dia 31 de janeiro de 2021. Todos os pacientes que foram internados em um dos três hospitais da Unimed Recife - Hospital Unimed Recife I, Hospital Unimed Recife III e Hospital Geral Unimed Recife (HGMI) - entraram no estudo. Nesse mesmo período avaliamos o número de pacientes com Covid-19 ou suspeita de Covid-19 que foram atendidos na emergência do Hospital Unimed Recife III.

O presente estudo foi baseado na Resolução número 466/12 do Conselho Nacional de Saúde referente a pesquisa com seres humanos a partir da assinatura da Carta de Anuência pela Direção da Instituição.

\section{Resultados}

Foram atendidos 126.553 pacientes na Unidade de Emergência do Hospital Unimed Recife III no período entre 26 de março de 2020 e 31 de janeiro de 2021, desses pacientes atendidos na emergência 39.340 (31,09\%) pacientes foram diagnosticados com tendo Covid-19 ou suspeita de Covid-19.

No período de 10 meses (26 março de 2020 até 31 de janeiro de 2021) foram internados 1.039 pacientes com Covid-19 em um dos três hospitais da Unimed Recife. A Figura 1 mostra o número de pacientes com Covid-19 internados por mês na Unimed Recife durante o período de março de 2020 até o fim de janeiro de 2021.

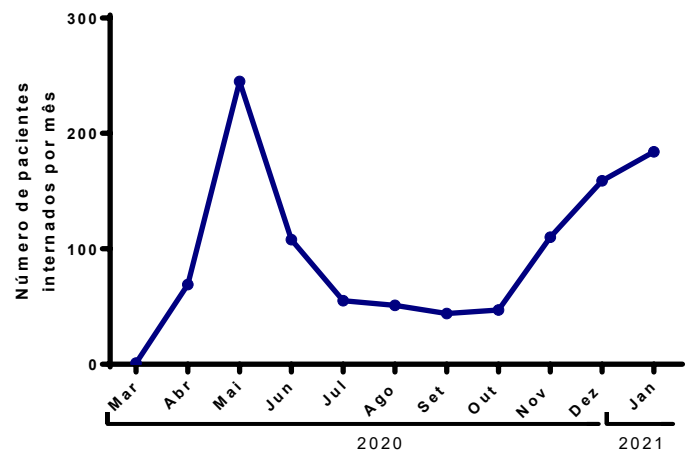

Figura 1. Número de pacientes com Covid-19 internados por mês na Unimed Recife. Perceber que a frequência no internamento acompanha aspectos epidemiológicos da doença no estado de Pernambuco, com um pico em maio de 2020 e outro em ascenção iniciando em novembro de 2020.

Todos esses 1.039 indivíduos receberam alta hospitalar ou foram a óbito. Foram 968 (93,2\%) pacientes clínicos e 71 $(6,8 \%)$ cirúrgicos. A Figura 2 mostra a distribuição dos pacientes de acordo com a faixa etária.

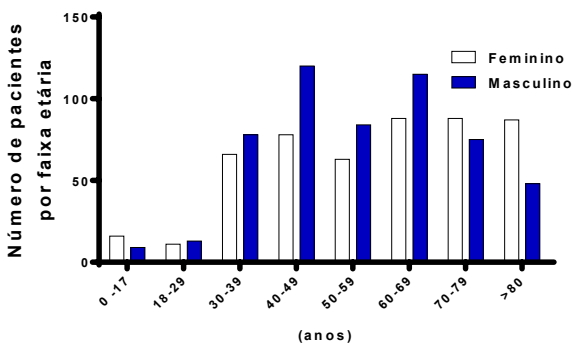

Figura 2. Distribuição dos 1.039 pacientes em relação ao sexo e faixa etária com Covid-19 internados em um dos três hospitais da Unimed Recife até 31 de janeiro de 2021. 
Na Tabela 1 são enumeradas as afecções associadas nos 1.039 pacientes com Covid-19. A média de permanência no hospital foi 11,2 dias (9,4 dias nos pacientes clínicos e 36,6 dias nos pacientes cirúrgicos). $O$ case mix dos pacientes clínicos foi 2,0293 e nos cirúrgicos 8,7278.

\section{Permanência média na ventilação mecânica}

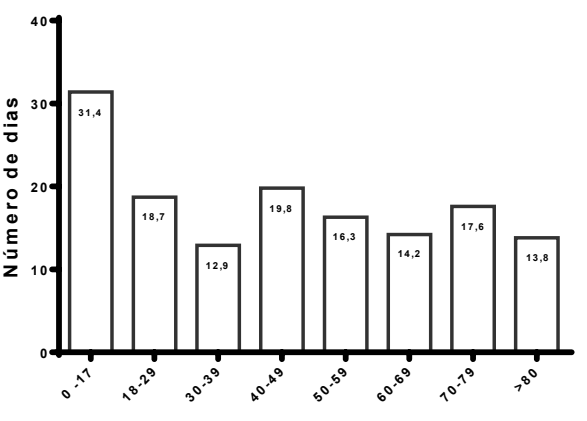

Faixa etária (anos)

Figura 3. Permanência média (em dias) na ventilação mecânica, de acordo com a faixa etária.

Foram internados na UTI 342/1.039 (32,9\%) dos pacientes e $57,9 \%$ deles utilizaram ventilação mecânica. Ventilação mecânica foi necessária em 198/1.039 (19,1\%) dos pacientes internados. A Figura 3 ilustra a permanência média na ventilação mecânica de acordo com a faixa etária. Letalidade por faixa etária entre os pacientes com Covid-19 que foram submetidos a ventilação mecânica é mostrada na Figura 4 .

Tabela 1. Presença de afecções associadas nos 1.039 pacientes com Covid-19

\begin{tabular}{|l|c|c|}
\hline \multicolumn{1}{|c|}{ Doenças associadas } & $\mathrm{n}$ & $\%$ \\
\hline Hipertensão arterial & 529 & 60,1 \\
\hline SARS & 281 & 31,9 \\
\hline Diabete melito & 264 & 30,0 \\
\hline Obesidade & 103 & 9,9 \\
\hline Insuficiência renal aguda & 79 & 10,0 \\
\hline Sepse & 68 & 7,7 \\
\hline Hipotiroidismo & 49 & 5,6 \\
\hline Tabagismo & 24 & 2,7 \\
\hline Insuficiência renal crônica & 23 & 2,6
\end{tabular}

SARS, Síndrome respiratória aguda grave.

Tabela 2. Letalidade (odds ratio e intervalo do confiança 95\%) observada por faixa etária nos 1.039 pacientes com Covid-19 internados nos três hospitais da Unimed Recife até 31 de janeiro de 2021

\begin{tabular}{c|c|c}
$\begin{array}{c}\text { Faixa etária } \\
\text { (anos) }\end{array}$ & $\begin{array}{c}\text { Odds Ratio (Intervalo de } \\
\text { confiança 95\%) }\end{array}$ & $\begin{array}{c}\text { Valor de } p \\
\text { (Teste exato de Fisher) }\end{array}$ \\
\hline
\end{tabular}

\begin{tabular}{|c|c|c|}
\hline $18-29$ & 1 & \\
\hline$<18$ & $0,31(0,01-7,92)$ & 0,490 \\
\hline $30-39$ & $1,00(0,12-8,70)$ & $>0,999$ \\
\hline $40-49$ & $0,12(0,01-1,93)$ & 0,205 \\
\hline $50-59$ & $2,42(0,300-19,320)$ & 0,698 \\
\hline $60-69$ & $3,83(0,498-29,51)$ & 0,216 \\
\hline $70-79$ & 7,73 (1,01 1-59,07) & 0,019 \\
\hline$\geq 80$ & $13,96(1,829-106,6)$ & $<0,001$ \\
\hline
\end{tabular}

A letalidade geral foi de 13,76\% (143 mortes/1.039 pacientes internados). Há um aumento na letalidade por Covid-19 com o aumento da idade (Figuras 5 e 6, e Tabela 2). Na Tabela 3 está registrado o número de mortes por período comparando-se os primeiros meses da pandemia (de março a agosto de 2020) versus os últimos meses (de setembro de 2020 até janeiro de 2021) entre os pacientes com Covid-19 internados na Unimed Recife. A letalidade no primeiro período da pandemia por Covid-19 foi significativamente maior, i.e., $17,6 \%$ versus $9,7 \%$.

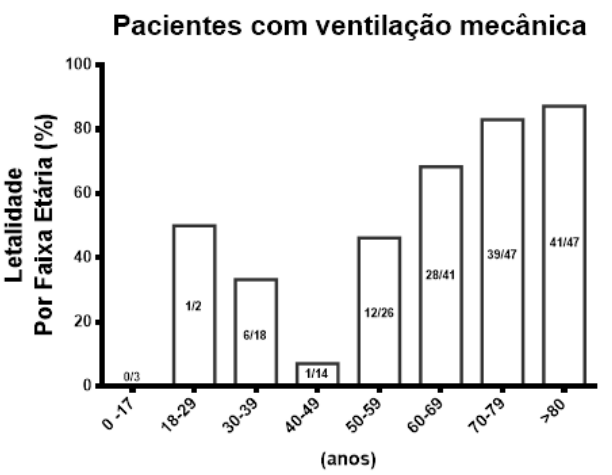

Figura 4. Letalidade entre os pacientes com Covid-19 que toram submetidos a ventilação mecânica por faixa etária.

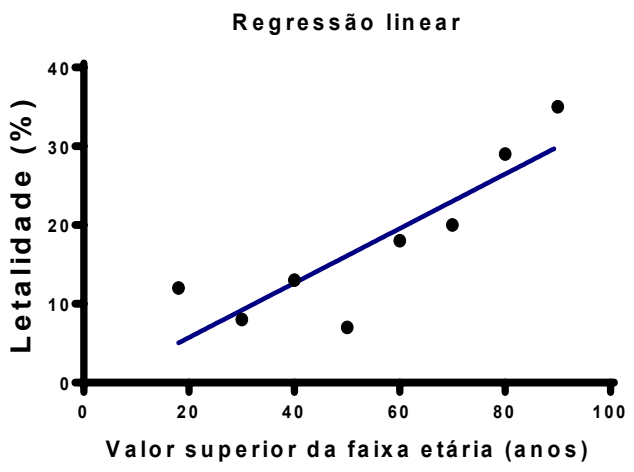

Figura 5. Regressão linear entre letalidade e faixa etária nos 1.039 pacientes com Covid-19. R2 0,07463; slope 0,34 \pm 0,08 (IC 95\% $0,01-0,55), p=0,006(F 17,65)$.

Obesidade aumentou significativamente a letalidade nos pacientes com Covid-19 [120 mortes/1.016 pacientes não obesos (11,8\%) versus 23 mortes/103 pacientes obesos $(22,3 \%)$, OR $2,15(1,30-3,50), p=0,005)]$.

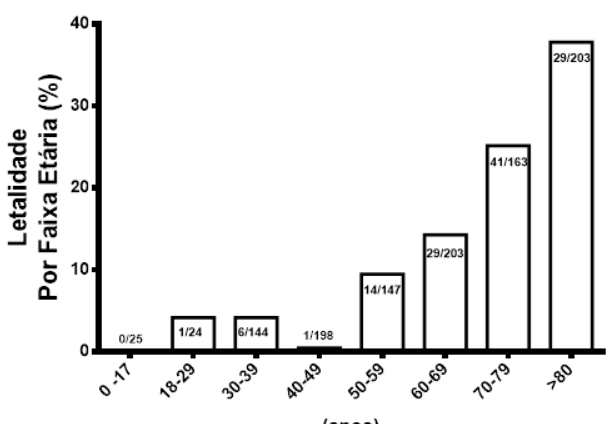

(anos)

Figura 6. Letalidade por Covid-19 nos pacientes internados na Unimed Recife de acordo com a faixa etária. 
Tabela 3. Número de mortes por período em comparação entre os primeiros meses da pandemia (de março a agosto de 2020) versus os últimos meses (de setembro de 2020 até janeiro de 2021) entre os pacientes com Covid-19 internados na Unimed Recife

\begin{tabular}{|c|c|c|c|c|}
\hline Período & $\begin{array}{c}\text { Número de } \\
\text { pacientes } \\
\text { internados }\end{array}$ & $\begin{array}{c}\text { Número de } \\
\text { mortes }\end{array}$ & OR (IC 95\%) & $\begin{array}{c}\text { Valor } \\
\text { de } p\end{array}$ \\
\hline $\begin{array}{l}\text { Março/2020 - } \\
\text { agosto/2020 }\end{array}$ & 534 & $94(17,6 \%)$ & $\begin{array}{c}1,99 \\
(1,37-2,88)\end{array}$ & $<0,001$ \\
$\begin{array}{l}\text { Setembro/2020 - } \\
\text { Janeiro/2021 }\end{array}$ & 505 & $49(9,7 \%)$ & & \\
\hline
\end{tabular}

\section{Discussão}

Neste estudo os autores estão avaliando a experiência da Unimed Recife, nos seus três hospitais, com o tratamento de pacientes que foram internados por Covid-19. Pelo fato do paciente ter sido internado em uma instituição hospitalar entende-se que há uma clara tendência de no estudo serem encontrados pacientes com o pior espectro de gravidade da doença. A maioria dos pacientes com Covid-19 foi atendida na emergência para o diagnóstico correto e devida orientação terapêutica sem a necessidade de um internamento. Também se percebe que a frequência no internamento ao longo do ano de 2020 apresenta um perfil semelhante aos aspectos epidemiológicos da doença no estado de Pernambuco, com um pico em maio de 2020 e outro em ascensão se iniciando em novembro de 2020.

Quando avaliamos letalidade em relação à idade, como esperado, foi significantemente maior nas faixas de idade mais avançadas e nos pacientes que necessitaram ventilação mecânica.

São vários os fatores associados com letalidade na Covid-19. ${ }^{19-25}$ Muitos dos pacientes que foram internados apresentavam comorbidades vinculadas com um pior prognóstico na Covid-19, como HAS (60\%), DM (30\%) e obesidade (10\%). ${ }^{21}$

Um dos primeiros estudos publicados sobre pacientes com Covid-19 avaliou 191 pacientes que foram internados em dois hospitais de Wuhan, China, ${ }^{26}$ até o dia 31 de janeiro de 2020. Dos 191 pacientes, 53 (28,3\%) morreram. Os autores relatam que 91 (48\%) dos pacientes apresentavam comorbidades, tais como: hipertensão arterial sistêmica (30\%), diabetes (19\%) e doença coronariana (8\%). O estudo mostrou após análise por regressão multivariável uma associação da idade mais avançada, escore elevado no SOFA (Sequential Organ Failure Assessment ) e nível do D-dímero elevado. ${ }^{26}$

No Brasil, a experiência do Hospital Sírio-Libanês, ${ }^{27}$ com 212 pacientes adultos com Covid-19, internados na UTI entre 8 de março de 2020 e 30 de junho de 2020, foi publicada e merece comentário. Os autores compararam esse grupo com outro grupo com 185 pacientes sem Covid-19 que foram internados na mesma UTI no ano anterior de 2019. Quando comparados aos controles históricos, os pacientes com Co- vid-19 necessitaram de mais suporte ventilatório, um maior tempo de ventilação mecânica e ficaram internados por maior número de dias na UTI e no hospital. Interessante o fato que não foi observada uma diferença estatística na letalidade. Foi também observada uma proporção maior de homens $(55,7 \%$ vs. $75,9 \%)$ e de obesidade mórbida $(7,5 \%$ versus $2,2 \%)$. Nessa série de pacientes com Covid-19 internados na UTI a letalidade foi de 9,0\% (16,2\% nos pacientes que receberam ventilação mecânica) aos 28 dias e 10,8\% (19,0\% nos pacientes que receberam ventilação mecânica) com 60 dias. Durante o internamento observou-se que 50,9\% usaram vasopressores, $49,5 \%$ foram submetidos a ventilação mecânica invasiva, 49,1\% com ventilação mecânica não invasiva com pressão positiva, 46,7\% receberam ventilação nasal de alto fluxo, 10,8\% dos pacientes foram traqueostomizados, 13,2\% receberam diálise renal, a duração da ventilação mecânica foi de 9 dias (6-16, mediana IQR). Sete dias foi a mediana na duração do internamento na UTI, quando o paciente necessitou de ventilação mecânica a duração foi de 9 dias (mediana).

Sabemos que a comparação de dados estatísticos entre diferentes instituições é impossível porque os critérios para admissão hospitalar ou na UTI são dististos, a população de pacientes também pode ter particularidades peculiares ao nível sócio-cultural e econômico.

Em um estudo transversal baseado em dados de 204 pacientes internados na UTI do Complexo Médico Hayatabad, no Paquistão, a letalidade geral foi de $77 \% .{ }^{28} \mathrm{~A}$ ventilação não invasiva foi usada por $61,8 \%$ dos pacientes. ${ }^{28} \mathrm{~A}$ letalidade foi maior para ventilação mecânica invasiva $(93,6 \%$ versus $66,7 \%, p<0,001)$ e nos pacientes com mais de 60 anos (87,3\% versus $72,3 \%, p=0,019)$. A letalidade sem comorbidades foi de $75,2 \%{ }^{28}$ Este estudo mostra uma letalidadealarmante nos pacientes com Covid-19 internados na UTI.

Outro estudo, este realizado na ltália ${ }^{7}$ com dados de 1.590 (64\% homens, $66 \pm 0,4$ anos) pacientes internados com Covid-19 em 26 hospitais, a hipertensão foi registrada em 54,9\% deles. A letalidade foi $11,8 \%$, apresentando esse grupo de pacientes $89,6 \pm 0,9$ anos de idade. ${ }^{7}$ Ao comparar o grupo de não sobreviventes com o grupo dos sobreviventes, observou-se uma maior frequência de idade avançada, hipertensão arterial, diabete melito, doença pulmonar obstrutiva crônica, doença renal crônica, doenças das artérias coronárias e insuficiência cardíaca. ${ }^{7} \mathrm{O}$ índice de comorbidade de Charlson foi significativamente maior nos não sobreviventes $(4,3 \pm 0,15$ versus $2,6 \pm 0,05) .^{7}$ Em relação aos fármacos, inibidores da ECA (enzima de conversão da angiotensina), diuréticos e beta-bloqueadores foram usados com mais frequência em pacientes do grupo não sobreviventes. Porém, após correção por análise multivariada, apenas a idade, diabete melito, doença pulmonar obstrutiva crônica e doença renal crônica, foram associadas com letalidade. 
Um estudo chinês, com 996 pacientes (282 com hipertensão arterial), teve o objetivo de avaliar o impacto da hipertensão na Covid-19 e verificar se o uso prévio de inibidores do sistema renina-angiotensina-aldosterona alteraria o prognóstico. ${ }^{29}$ Interessante foi o fato que os pacientes com Covid-19 com hipertensão tinham infecções secundárias mais graves, anormalidades cardíacas e renais e depleção de células CD8 (+) na admissão. Os pacientes hipertensos com história de tratamento com inibidor do sistema renina-angiotensina-aldosterona tinham níveis mais baixos de proteína $C$ reativa e níveis mais altos de células CD4 (+). A letalidade foi menor nos pacientes do grupo com inibidor (9,8\% versus $26,1 \%)$. Os autores concluíram que a hipertensão pode ser um fator de risco independente para todas as causas de letalidade nos pacientes com Covid-19 e aqueles que anteriormente usaram inibidores do sistema renina-angiotensina-aldosterona apresentaram um melhor prognóstico.

Em relação a idade como fator de risco, um estudo realizado no México com 101.728 pacientes com Covid-19, mostrou que no grupo de 20.804 pacientes com idade $\geq 60$ anos (20.5\%) a letalidade foi maior $(6,95 \%$ versus $29,49 \%) .{ }^{13}$

Preocupados em saber a taxa de letalidade (independente se o paciente estava internado em um hospital ou não) por Covid-19 em Rondônia, Brasil, os pesquisadores verificaram que 1.020 (letalidade de 2, 1\%) de 49.804 pacientes com Covid-19 morreram. ${ }^{14}$ Houve uma maior taxa de letalidade entre os pacientes com $\geq 80$ anos de idade $(24,89 \%)$ quando comparada com paciente com 20-39 anos de idade (0,34\%). ${ }^{14}$

Os Diagnosis Related Groups (DRG) faz parte de um sistema de classificação de pacientes desenvolvida na Universidade de Yale, nos Estados Unidos, de forma que servem de instrumento a gestão de um hospital, permitindo mensuração e avaliação do desempenho. ${ }^{30}$ É uma ferramenta versátil e muito importante também para pesquisa porque guarda com eficiência dados dos pacientes internados em um hospital, permitindo comparações. Os dados usados no presente estudo foram obtidos de uma plataforma DRG.

Como case mix entende-se a proporção relativa de diferentes tipos de pacientes que recebem tratamento no hospital. ${ }^{30}$ Carnielo ${ }^{31}$ comenta e define o termo case mix como "uma medida de complexidade e criticidade hospitalar baseado na idade, doença que determinou a internação, nas doenças pré-existentes e nos procedimentos realizados, usado em todo o mundo desde a década de 80 e mensurado pela metodologia DRG." No nosso estudo o case mix dos pacientes com Covid-19 cirúrgico foi mais elevado em ralação aos pacientes clínicos.

Concluímos que a Covid-19 é uma doença com prognóstico reservado, principalmente naqueles pacientes idosos, com diabete melito, hipertensão arterial e obesidade. Na segunda fase da pandemia por Covid-19 percebemos uma queda signi- ficativa na letalidade por Covid-19 nos pacientes internados. Covid-19 é uma doença nova, ainda pouco conhecida, e nos primeiros meses não se entendia muito bem sobre os mecanismos pelos quais o vírus se multiplicava nem como o processo fisiopatológico ocorria. Muito se aprendeu sobre a doença nos últimos meses, provavelmente a diminuição na letalidade é o resultado de estudos clínicos sobre a melhor forma de se tratar a Covid-19. Neste período de março de 2021 até fevereiro de 2021 foram seis os protocolos clínicos desenvolvidos e ou atualizados na Unimed Recife relacionados com o tratamento do paciente com Covid-19, mostrando as mudanças de conduta para com um paciente grave com essa doença com alta taxa de letalidade.

Conflito de interesse Não há conflito de interesses.

Financiamento Os autores não receberam financiamento específico para este trabalho.

Contribuições do autor MMV, FC Conceito e desenho do estudo; MMV, FC Coleta de Dados; MMV Análise e interpretação de dados; MMV Redação do manuscrito; FC, MMV Revisando para conteúdo intelectual; MMRFF, CAB, AMGS, DFN, AO contribuíram com dados ou ferramentas de análise; MMV, MMRFFF, CAB, AMGS, DFN, ACCCA, FC, AO Aprovação final do manuscrito.

Marcelo Moraes Valença

https://orcid.org/0000-0003-0678-3782

Martha Maria Romeiro Figueiroa F. Fonseca

https://orcid.org/0000-0003-4929-6343

Cátia Arcuri Branco

https://orcid.org/0000-0002-7082-9223

Alex Maurício Garcia Santos

https://orcid.org/ 0000-0002-7472-041X

Antonio Oliveira

https://orcid.org/0000-0003-4368-5305

Danilo Ferreira Nunes

https://orcid.org/

Ana Carolina Chiappetta Correia de Araújo

https://orcid.org/

Fernando Cruz

https://orcid.org/0000-0002-6152-6888

\section{References}

1. Tang Y, Serdan TDA, Masi LN, Tang S, Gorjao R, Hirabara SM. Epidemiology of COVID-19 in Brazil: using a mathematical model to estimate the outbreak peak and temporal evolution. Emerg Microbes Infect. 2020;9: 1453 1456.

2. Souza WV, Martelli CMT, Silva A, et al. The first hundred days of COVID-19 in Pernambuco State, Brazil: epidemiology in historical context. Cad Saude Publica. 2020;36:e00228220.

3. Goossens GH, Dicker D, Farpour-Lambert NJ, et al. Obesity and COVID-19: A Perspective from the European Association for the Study of Obesity on Immunological Perturbations, Therapeutic Challenges, and Opportunities in Obesity. Obes Facts. 2020;13:439-452.

4. Aghili SMM, Ebrahimpur M, Arjmand B, et al. Obesity in COVID-19 era, implications for mechanisms, comorbidities, and prognosis: a review and meta-analysis. Int J Obes (Lond). 2021. 
5. Zhou Y, Chi J, Lv W, Wang Y. Obesity and diabetes as high-risk factors for severe coronavirus disease 2019 (Covid-19). Diabetes Metab Res Rev. 2021;37:e3377.

6. Sanchis-Gomar F, Lavie CJ, Mehra MR, Henry BM, Lippi G. Obesity and Outcomes in COVID-19: When an Epidemic and Pandemic Collide. Mayo Clin Proc. 2020;95:1445-1453.

7. laccarino G, Grassi G, Borghi C, Ferri C, Salvetti M, Volpe M. Age and Multimorbidity Predict Death Among COVID-19 Patients: Results of the SARS-RAS Study of the Italian Society of Hypertension. Hypertension. 2020;76:366-372.

8. Kamyshnyi A, Krynytska I, Matskevych V, Marushchak M, Lushchak O. Arterial Hypertension as a Risk Comorbidity Associated with COVID-19 Pathology. Int J Hypertens. 2020;2020:8019360.

9. Shah H, Khan MSH, Dhurandhar NV, Hegde V. The triumvirate: why hypertension, obesity, and diabetes are risk factors for adverse effects in patients with COVID-19. Acta Diabetol. 2021.

10. $\mathrm{Xu} P \mathrm{PP}$, Tian RH, Luo $\mathrm{S}$, et al. Risk factors for adverse clinical outcomes with COVID-19 in China: a multicenter, retrospective, observational study. Theranostics. 2020; 10:6372-6383.

11. Alamdari NM, Afaghi S, Rahimi FS, et al. Mortality Risk Factors among Hospitalized COVID-19 Patients in a Major Referral Center in Iran. Tohoku J Exp Med. 2020;252:73-84.

12. Dai Z, Zeng D, Cui D, et al. Prediction of COVID-19 Patients at High Risk of Progression to Severe Disease. Front Public Health. 2020;8:574915.

13. Bello-Chavolla OY, Gonzalez-Diaz A, Antonio-Villa NE, et al. Unequal Impact of Structural Health Determinants and Comorbidity on COVID-19 Severity and Lethality in Older Mexican Adults: Considerations Beyond Chronological Aging. J Gerontol A Biol Sci Med Sci. 2021;76:e52-e59.

14. Escobar AL, Rodriguez TDM, Monteiro JC. Lethality and characteristics of deaths due to COVID-19 in Rondonia: an observational study. Epidemiol Serv Saude. 2020;30:e2020763.

15. Ebell MH, Cai X, Lennon $R$, et al. Development and Validation of the COVID-NoLab and COVID-SimpleLab Risk Scores for Prognosis in 6 US Health Systems. J Am Board Fam Med. 2021;34:S127-S 135.

16. Brandi ML, Giustina A. Sexual Dimorphism of Coronavirus 19 Morbidity and Lethality. Trends Endocrinol Metab. 2020;31:918-927.

17. Fu L, Wang B, Yuan T, et al. Clinical characteristics of coronavirus disease 2019 (COVID-19) in China: A systematic review and meta-analysis. JInfect. 2020;80:656-665.

18. Moutchia J, Pokharel P, Kerri A, et al. Clinical laboratory parameters associated with severe or critical novel coro navirus disease 2019 (COVID-19): A systematic review and meta-analysis. PLoS One. 2020;15:e0239802.

19. Alamer AA, Almulhim AS, Alrashed AA, Abraham I. Mortality, Severity, and Hospital Admission among COVID-19 Patients with ACEI/ARB Use: A Meta-Analysis Stratifying Countries Based on Response to the First Wave of the Pandemic. Healthcare (Basel). 2021;9.

20. Solano-Lopez J, Zamorano JL, Pardo Sanz A, et al. [Risk factors for in-hospital mortality in patients with acute myocardial infarction during the COVID-19 outbreak]. Rev Esp Cardiol. 2020;73:985-993.

21. Rastad H, Karim H, Ejtahed HS, et al. Risk and predictors of in-hospital mortality from COVID-19 in patients with diabetes and cardiovascular disease. Diabetol Metab Syndr. 2020; 12:57.

22. Rieg S, von Cube $M$, Kalbhenn J, et al. COVID-19 in-hospital mortality and mode of death in a dynamic and non-restricted tertiary care model in Germany. PLOS One. 2020; 15:e0242127.

23. Nie L, Dai K, Wu J, et al. Clinical characteristics and risk factors for in-hospital mortality of lung cancer patients with COVID-19: A multicenter, retrospective, cohort study. Thorac Cancer. 2021; 12:57-65.

24. Janke AT, Mei H, Rothenberg C, Becher RD, Lin Z, Venkatesh AK. Analysis of Hospital Resource Availability and COVID-19 Mortality Across the United States. J Hosp Med. 2021.

25. Zhang G, An Y, Zhang L, Xie L, Guo X. Risk factors for in-hospital mortality in patients with cancer and COVID-19. Lancet Oncol. 2020;21:e407.

26. Zhou $F, Y u T, D u R$, et al. Clinical course and risk factors for mortality of adult inpatients with COVID-19 in Wuhan, China: a retrospective cohort study. Lancet. 2020;395:1054-1062.

27. Socolovithc RL, Fumis RRL, Tomazini BM, et al. Epidemiology, outcomes, and the use of intensive care unit resources of critically ill patients diagnosed with COVID-19 in Sao Paulo, Brazil: A cohort study. PLoS One. 2020; 15:e0243269.

28. Rahim F, Amin S, Noor M, et al. Mortality of Patients With Severe COVID-19 in the Intensive Care Unit: An Observational Study From a Major COVID-19 Receiving Hospital. Cureus. 2020; 12:e10906.

29. Pan W, Zhang J, Wang $M$, et al. Clinical Features of COVID-19 in Patients With Essential Hypertension and the Impacts of Renin-angiotensin-aldosterone System Inhibitors on the Prognosis of COVID-19 Patients. Hypertension. 2020;76:732-741.

30. Noronha MF, Portela MC, Lebrao ML. [Potential uses of AP-DRG to describe the health care profile in hospital units]. Cad Saude Publica. 2004;20 Suppl 2:S242-255.

31. Carnielo M. Especialista pontua os desafios da saúde pós-pandemia. In. Portal Hospitais Brasil; 2021. 